\title{
Treat-to-Target bei systemischem Lupus erythematodes
}

\author{
Martin Aringer ${ }^{a} \quad$ Nicolai Leuchten ${ }^{a} \quad$ Matthias Schneider ${ }^{b}$ \\ a Bereich Rheumatologie, Medizinische Klinik und Poliklinik III, Universitätsklinikum und Medizinische Fakultät \\ Carl Gustav Carus der TU Dresden, Dresden, Deutschland; \\ ${ }^{b}$ Abteilung für Rheumatologie, Heinrich-Heine-Universität, Düsseldorf, Deutschland
}

\author{
Schlüsselwörter \\ Systemischer Lupus erythematodes · Remission · Lupus Low \\ Disease Activity · Glukokortikoide · Hydroxychloroquin . \\ Schub $\cdot$ Schaden
}

\section{Zusammenfassung}

Das Treat-to-target-Prinzip, bei dem die entzündliche Krankheitsaktivität mithilfe von krankheitsmodifizierenden Arzneimitteln (disease modifying anti-rheumatic drugs, DMARDs) oder Immunsuppressiva unter Kontrolle gebracht wird, ist auch für den systemischen Lupus erythematodes (SLE) umzusetzen. Eine Intensivierung der Immunsuppression mit höher dosierten Glukokortikoiden kann die Behandlungsergebnisse bei SLE jedoch verschlechtern. Infolgedessen sprechen sich alle aktuellen Empfehlungen für eine bessere Krankheitskontrolle bei gleichzeitiger Begrenzung der täglichen Glukokortikoid-Dosen auf maximal 5 oder 7,5 mg PrednisoIon aus. Zusätzlich erfolgen eine Behandlung mit Hydroxychloroquin und weitere prophylaktische Maßnahmen, und das AntiPhospholipid-Syndrom wird mit Antikoagulantien und nicht mit Immunsuppressiva behandelt. Dies macht den Treat-to-target-Therapieansatz beim SLE etwas schwieriger, spiegelt jedoch die Komplexität der Erkrankung wider. @ 2019 S. Karger GmbH, Freiburg
Bei vielen systemischen Autoimmunerkrankungen haben die Betroffenen vergleichbare Probleme und die wichtigsten übergeordneten Therapieprinzipien sowie viele Behandlungsansätze sind ähnlich. Dies gilt nicht nur für die häufigeren Formen der Arthritis und der Erkrankungen des Achsenskeletts, sondern auch für die klassischen systemischen Autoimmunerkrankungen wie den systemischen Lupus erythematodes (SLE). Aufgrund der höheren Komplexität treten beim SLE jedoch zusätzliche Hindernisse für einen systematischen Ansatz auf, sei es in Hinblick auf die Klassifizierung [1], die angestrebte Remission [2] oder die zielgerichtete Therapie (treat to target, T2T) [3].

Was die rheumatischen Erkrankungen betrifft, wurde das T2TKonzept zuerst in der Behandlung der Rheumatoiden Arthritis (RA) erfolgreich etabliert [4] und hat seither die RA-Therapie wesentlich beeinflusst. Es hat nicht nur dazu beitragen, die Ergebnisse zu verbessern, sondern hat auch als Blaupause für die Stan- dardisierung der RA-Therapie gedient [5]. Ein ähnlicher Ansatz für den SLE wäre daher sehr wünschenswert. Tatsächlich veröffentlichte eine internationale Arbeitsgruppe unter Dr. van Vollenhoven 2014 erste T2T-Empfehlungen für den SLE [3]. Wie realistisch ist dieser Ansatz und wie weit sind wir bis heute gekommen?

\section{SLE-spezifische Aspekte}

Der SLE unterscheidet sich in verschiedenen Punkten von der RA, was den T2T-Ansatz erschwert. Einer der Unterschiede besteht darin, dass der SLE nicht durch eine alleinige Hauptorganmanifestation gekennzeichnet ist. Die Symptome beim SLE sind zahlreich und von unterschiedlicher prognostischer Relevanz. Sie reichen von Gelenkbeteiligung und mukokutanen Manifestationen über verschiedene Zytopenien, verschiedene Formen der Immunkomplex-Nephritis oder Vaskulitis bis hin zu einer neuropsychia- information@karger.com

www.karger.com/kai 
trischen Beteiligung und thrombotischen Ereignissen durch AntiPhospholipid-Antikörper und erfordern individuelle Behandlungsmodalitäten [6, 7]. Diese Vielfalt mit einer einzigen Messung der Krankheitsaktivität zu erfassen, stellt eine Herausforderung dar, und die damit verbundene Unsicherheit wird durch die relativ hohe Zahl an konkurrierenden Messinstrumenten zur Ermittlung der SLE-Krankheitsaktivität widergespiegelt [8-11].

Einige Organmanifestationen, wie die schwere Lupusnephritis oder die ZNS-Vaskulitis, führen häufig zu schweren Organschäden und können sogar lebensbedrohlich sein [12-15]. Tatsächlich ist der Schaden beim SLE mit einer Zunahme der Schäden und mit einem höheren Mortalitätsrisiko verbunden [16, 17]: «Da der Schaden ein Prädiktor für Folgeschäden und Tod ist, sollte die Verhinderung zusätzlicher Schäden ein wichtiges therapeutisches Ziel bei SLE sein», so die T2T-Empfehlungen für den SLE [3]. Der Schaden ist in der Regel durch entzündliche Manifestationen bedingt, die zudem eine der Hauptursachen [18] für das stark erhöhte Atherosklerose- und Mortalitätsrisiko sind [19]. Der andere treibende Faktor ist die hoch dosierte Glukokortikoidtherapie [20], wobei Glukokortikoide jedoch weiterhin einen Grundpfeiler der Therapie bilden. Daher sind die Variabilität der Erkrankung sowie die potenziellen negativen Auswirkungen einiger Therapeutika Hindernisse auf dem Weg zu einem T2T-Ansatz bei SLE.

\section{Definition der Therapieziele}

Der erste Schritt beim T2T-Ansatz besteht in der Festlegung langfristiger, sinnvoller Ziele. Die T2T-Empfehlungen für den SLE geben als übergeordnetes Prinzip an: «Die Behandlung des SLE sollte darauf abzielen, das langfristige Überleben zu sichern, Organschäden zu verhindern und die gesundheitsbezogene Lebensqualität zu verbessern» [3].

Diese übergeordneten Ziele entsprechen in gewisser Weise einem Flugzeug, das seine Passagiere glücklich am Zielflughafen entlässt. Hingegen gleicht der T2T-Ansatz vielmehr dem Instrumentensystem, das im Landeanflug anzeigt, ob sich die Maschine noch auf dem richtigen Weg zum optimalen Aufsetzpunkt auf der Landebahn befindet. Die Idee dahinter ist, etwaige Abweichungen von der optimalen Bahn rechtzeitig zu korrigieren, um sicherzustellen, dass das Ziel erreicht wird. Diese Korrekturfunktion basiert im Wesentlichen auf objektiven und wiederholt gemessenen Parametern, die nicht nur die Tatsache einer Abweichung als solche anzeigen, sondern auch die Richtung und das Ausmaß der erforderlichen Gegenmaßnahmen.

Das oben erwähnte übergeordnete Prinzip mündet in einen ganz konkreten Ansatz, nämlich «Kontrolle der Krankheitsaktivität und Minimierung von Komorbiditäten und Arzneimitteltoxizität» [3]. Allerdings ist diese Empfehlung nicht von unmittelbarem Nutzen. Dafür ist in erster Linie ein erreichbares, kurz- bis mittelfristiges Ziel erforderlich [21]. Bei der RA ist die Remission (oder eine geringe Krankheitsaktivität, wenn eine Remission nicht zu erreichen ist) - um im Bild des Flugzeugs zu bleiben - als der richtige Weg zur Landung definiert [5]; doch auch Scores wie der Clinical Disease Activity Index (CDAI) und der Simple Di- sease Activity Index (SDAI) liefern ein Maß für den Abstand zu diesem Zielpunkt, der Einfluss auf die optimale Vorgehensweise für das Erreichen einer ausreichenden Kontrolle der Krankheitsaktivität hat.

Es ist folgerichtig, auch beim SLE eine Remission oder geringe Krankheitsaktivität als kurzfristiges Ziel für einen T2T-Ansatz anzustreben. Tatsächlich wurde die Remission in den internationalen T2T-Empfehlungen für den SLE als Ziel festgelegt [3]. Eine allgemein akzeptierte Definition der Remission bei SLE liegt bisher jedoch nicht vor. Zwar ist die Situation ähnlich wie in den Anfängen des T2T-Ansatzes bei RA, als ebenfalls noch keine solche Definition existierte, doch besteht ein weiterer Unterschied: Denn damals war klar, dass Remission das Fehlen einer aktiven Arthritis bedeutete [4]. Beim SLE ist dies nicht so einfach. Eine Remission muss die Abwesenheit einer aktiven Gelenkerkrankung, einer aktiven Hautkrankheit oder einer aktiven Nierenerkrankung beinhalten. Darüber hinaus muss sie auch das Fehlen einer hämolytischen Anämie einschließen. Andere Maße erfordern jedoch unter Umständen eine unterschiedliche Behandlung. So haben beispielsweise Leukopenie, Lymphopenie oder Thrombozytopenie nur dann ungünstige Folgen, wenn sie ein bestimmtes Ausmaß erreichen, wie etwa bei einer schweren Thrombozytopenie. Leichte Veränderungen rechtfertigen möglicherweise keine Steigerung der Therapie, und ein etwaiges Anti-Phospholipid-Syndrom (APS) erfordert die Gabe von Antikoagulantien und keine Immunmodulation.

Die Remission beim SLE muss das Fehlen aller entzündlichen Manifestationen beinhalten. Dies ist entscheidend, um Organschäden zu vermeiden, wie etwa an den Gelenken (Jaccoud-ähnliche Arthropathie), der Haut (Narben nach Läsionen des diskoiden Lupus erythematodes (DLE)), den Nieren (Niereninsuffizienz) oder der Lunge (Fibrose). Daneben ist die vorzeitige Atherosklerose zu einem Hauptproblem bei lange bestehendem SLE geworden, was Studien zufolge mit der inflammatorischen Krankheitsaktivität zusammenhängt. Aus diesem Grund würde auch eine Anämie bei chronischer Erkrankung als weiteres Zeichen einer anhaltenden Entzündung eine Remission ausschließen. Hämolytische Anämie und schwere Leukopenie oder Thrombozytopenie müssen wegen ihrer Behandlungsbedürftigkeit ebenfalls ausgeschlossen werden. Dieser Teil ist unkompliziert.

Es gibt jedoch auch eine Untergruppe von Patienten, die an verschiedenen systemischen Symptomen mit oder ohne möglichen Zusammenhang mit der Krankheitsaktivität leiden. Fieber gehört immer dazu, wohingegen Arthralgien und Myalgien verschiedene Ursachen haben können. Noch schwieriger ist die Beurteilung der Fatigue, die eines der Hauptsymptome von SLE-Patienten darstellt. Fatigue kann durch einen Schaden oder durch Fibromyalgie, Depression oder eine Vielzahl von nicht SLE-bezogenen Problemen bedingt sein, aber auch durch die SLE-Krankheitsaktivität. Fatigue ist bei SLE häufig und nicht als Bagatellsymptom anzusehen [22, 23]. Die Patienten klagen nicht über eine leichte Müdigkeit, sondern über eine extreme Erschöpfung, die die meisten gesunden Menschen nur als Zustand kurz vor Ausbruch einer Grippe oder anderer fieberhafter Viruserkrankungen kennen. Schon das Hoch-
46

Kompass Autoimmun 2020;2:45-51 DOI: $10.1159 / 000507644$ 
steigen weniger Stufen fällt den Betroffenen in diesem Zustand schwer und am liebsten möchten sie sich hinlegen. Es ist sehr wahrscheinlich, dass zwischen einer Virusinfektion und dem aktiven SLE Ähnlichkeiten bestehen, denn beide führen zur Bildung von Immunkomplexen und zur Freisetzung von Interferon. Trotzdem galt Fatigue häufig nicht als mit der Krankheitsaktivität assoziiert. Dies hat sich teilweise geändert, seit in den Studien zu Belimumab eine deutliche Verbesserung der SLE-bedingten Fatigue nachgewiesen wurde [24]. Allerdings ist dadurch die Zuschreibung zur Krankheitsaktivität nicht einfacher geworden. Wenn keine erkennbaren entzündlichen Organmanifestationen nachweisbar sind, erfolgt die Zuschreibung der Fatigue zur SLE-Krankheitsaktivität heutzutage oft auf Grundlage der SLE-Serologie.

In Ermangelung besserer Instrumente ist dies derzeit die bestmögliche Einschätzung. Bislang wurde jedoch kein eindeutiger und einfacher pathophysiologischer Zusammenhang zwischen niedrigen Komplement- und/oder hohen Autoantikörper-Spiegeln und Fatigue nachgewiesen. Zudem ist eine aktive Serologie nicht notwendigerweise mit einer aktiven Erkrankung oder einer Zunahme des Langzeitschadens verbunden. Tatsächlich zeigte eine Untergruppe von Patienten mit aktiver Serologie und klinisch ruhendem SLE über 10 Jahre einen sehr guten Verlauf [25]. Daher stellen die ersten T2T-Empfehlungen für SLE ausdrücklich fest: «Es wird nicht empfohlen, die Therapie bei klinisch asymptomatischen Patienten allein auf Grundlage einer stabilen oder persistierenden serologischen Aktivität zu steigern» [3]. Da die Evidenzlage nicht eindeutig ist, ist es unsicher anzunehmen, eine aktive Serologie sei bei SLE-Patienten mit Fatigue mit einer aktiven Erkrankung gleichzusetzen. In jedem Fall sollte eine etwaige zusätzliche immunmodulatorische oder immunsuppressive Therapie einer erneuten Beurteilung unterzogen werden, wenn keine Besserung der Fatigue eintritt.

Alle diese Einzelheiten erschweren die Entwicklung einer praktikablen Definition von «Remission». Ronald van Vollenhoven und seine internationale Arbeitsgruppe kamen zu einer vorläufigen Definition, der zufolge das «Behandlungsziel bei SLE die Remission systemischer Symptome und Organmanifestationen oder, wenn eine Remission nicht erreichbar ist, die niedrigstmögliche Krankheitsaktivität gemäß einem validierten Lupus-Aktivitätsindex und/oder organspezifischen Markern» sein sollte [3]. Der letzte Teil ist besonders wichtig, da die validierten SLE-Aktivitätsscores alle einem dichotomen Ansatz für Manifestationen wie Arthritis oder Nephritis folgen: Bei Arthritis zählen 28 geschwollene Gelenke genauso viel wie 2, und bei Proteinurie 6,5 g pro Tag genauso viel wie $0,7 \mathrm{~g}$ pro Tag.

Daher sind organspezifische Maße bei der Versorgung von Patienten mit SLE von großer Bedeutung. Das Ziel muss sein, die Zahl der aktiv entzündeten Gelenke auf 0 zu reduzieren, was bei allen Standard-Maßen der SLE-Aktivität als 0 zählen würde, und ein aktives Gelenk, das in den meisten Scores noch unberücksichtigt bliebe, kommt diesem Ziel nahe. Bevor das erreicht ist, erscheint es jedoch erfoderlich, dass die Zahl der betroffenen Gelenke ermittelt wird, um festzustellen, ob es zu einer Verbesserung kommt, die sich später hoffentlich in einer Gelenkremission äu- ßert. Dasselbe gilt für die mukokutane Erkrankung, bei der der Revised Cutaneous Lupus Erythematosus Disease Area and Severity Index (R-CLASI) hilfreich sein kann, um eine Verbesserung zu messen. Bei Proteinurie dagegen lassen die Standardscores nicht mehr als $0,4 \mathrm{~g}$ oder $0,5 \mathrm{~g}$ pro Tag zu. Angesichts der Tatsache, dass Daten zufolge $0,7 \mathrm{~g}-0,8 \mathrm{~g}$ pro Tag oder weniger zu guten Langzeitergebnissen führen, könnte dies zu streng sein, wohingegen die alleinige Erfassung eines Anstiegs, wie beim SELENASLEDAI (Safety of Estrogens in Systemic Lupus Erythematosus National Assessment - Systemic Lupus Erythematosus Disease Activity Index) zu einer Unterschätzung der bestehenden Krankheit führen könnte.

Die Bemühungen der Asia-Pacific Lupus Collaboration Group unter Eric Morand, ein weniger strenges Konzept als die «Remission» zu entwickeln, führten zur Definition des Begriffs «Lupus low disease activity state» (LLDAS) [26]. Dr. Morand und seine Kollegen argumentieren überzeugend, dass der aktive SLE sehr variabel ist, während ein inaktiver SLE viele Ähnlichkeiten aufweist. Ihre Definition basiert hauptsächlich auf dem SLE-Krankheitsaktivitätsindex (SLEDAI) und lässt dabei einen Wert bis $4 \mathrm{zu}$, jedoch ohne aktive Beteiligung von Nieren, ZNS, Herz oder Lunge, ohne aktive Vaskulitis, ohne bestehende Hämolyse und ohne neu aufgetretene Symptome. Um auch Organsymptome, die nur gelegentlich auftreten und die im SLEDAI nicht abgebildet sind, vollständig darzustellen, begrenzt die Definition zudem die ärztliche Gesamtbeurteilung auf $\leq 1$ auf der visuellen Analogskala (VAS 0-3) und erlaubt nur gut verträgliche, zugelassene Medikamente und Glukokortikoide in Dosierungen von bis zu 7,5 mg Prednis(ol)onäquivalent pro Tag. Zwar handelt es sich in diesem Fall nicht um eine Remission, da beispielsweise eine aktive Gelenkbeteiligung zu weiterem Schaden führen könnte, doch ist diese Definition mit deutlich verbesserten Langzeitergebnissen verbunden und unterscheidet in randomisierten kontrollierten SLEStudien zwischen Wirkstoff und Placebo [26-28].

Diese Definition bildete einen hilfreichen Hintergrund für die Arbeit der Task Force «Definition of Remission in SLE» (DORIS), die ebenfalls von Ronald van Vollenhoven zusammengebracht wurde [2]. Diese Aufgabe ist schwierig und noch nicht abgeschlossen. Es gibt jedoch Elemente der Arbeitsdefinition von «Remission», über die sich alle Beteiligten einig sind. Während beim LLDAS ein SLEDAI von bis zu 4 akzeptabel ist, stützt sich die Definition der Remission auf einen klinischen SLEDAI von 0 oder, in der ersten Version, auf einen klinischen ECLAM (European Consensus Lupus Activity Measure) oder einen BILAG (British Isles Lupus Assessment Group)-Index von 0. Dabei wird die Untergruppe von Patienten mit serologisch aktivem, klinisch ruhendem SLE berücksichtigt. Während die ärztliche Gesamtbeurteilung auf der VAS von 0-3 beim LLDAS 1 betragen kann, liegt der obere Grenzwert gemäß der Remissionsdefinition bei $<0,5$, und selbst die mildeste Version der DORIS-Remission begrenzt die Glukokortikoide auf $5 \mathrm{mg}$ Prednis(lo)on pro Tag [2].

Mittlerweile liegen Hinweise vor, nach denen ein Zustand geringer Krankheitsaktivität für viele Patienten realistisch und - zumindest kurz- bis mittelfristig - mit besseren Behandlungsergeb- 
nissen verbunden ist [26]. Eine Remission ist eindeutig schwieriger zu erreichen [29-31]. Erste Studien sprechen jedoch für einen zusätzlichen Nutzen [29], wobei allerdings nicht alle Gruppen dem zustimmen. Somit gibt es zumindest einen vorläufigen Korridor für unseren T2T-Ansatz, bei dem der LLDAS die breitere Zielsetzung definiert und die Remission das engere, genauere Ziel, das möglicherweise nicht für jeden Patienten erreichbar ist.

\section{Behandlung und Remission}

Darüber, ob jegliche Therapie zu akzeptieren ist, wenn es um Remission geht, wurde sehr kontrovers diskutiert. Dies gilt nicht für Hydroxychloroquin, das nach Ansicht der meisten Experten weltweit heute von jedem SLE-Patienten eingenommen werden sollte, sofern keine Kontraindikationen bestehen [3, 14, 32]. Dagegen ist die Frage, ob das Konzept der Remission auch mit sehr niedrigen Dosierungen von Glukokortikoiden, Immunmodulatoren oder Immunsuppressiva vereinbar ist, Gegenstand von Diskussionen [2, 26]. Die Argumentation, nach der diese Medikamente erlaubt werden sollten, berücksichtigt die derzeitige Realität, in der eine Heilung in den meisten Fällen noch nicht realistisch ist [33] und eine Remission bei anderen Autoimmunerkrankungen wie RA durch die Langzeitgabe von krankheitsmodifizierenden Antirheumatika (DMARDs) erreicht wird [5].

Beim T2T-Ansatz überwiegen diese Argumente. In der Tat war sich die DORIS-Gruppe einig, dass eine Remission keine Heilung darstellt. Auf der anderen Seite würden die meisten Patienten eindeutig eine Remission ohne Glukokortikoide, DMARDs oder immunsuppressive Therapie bevorzugen. Die Gruppe der Patienten, die dieses hohe Ziel erreicht, scheint jedoch zu klein zu sein, als dass dies derzeit ein hilfreiches T2T-Konzept wäre [34]. Daher enthält die erste Konsensusversion zur Remission eine «Remission unter Therapie» und eine «Remission ohne Therapie», wobei bei letzterer trotzdem eine Hydroxychloroquin-Monotherapie gestattet ist.

Die «Remission unter Therapie» und der LLDAS schließen weiter eine Hochrisikotherapie wie etwa Cyclophosphamid aus [26]. Diese sollte zeitlich und bezüglich der Dosis streng begrenzt und nur bei organbedrohenden oder lebensbedrohlichen Erkrankungen eingesetzt werden, da kumulative Dosen $>30$ g das Risiko für akute myeloische Leukämie und wahrscheinlich auch für andere maligne Erkrankungen stark erhöhen, während es bereits unter sehr viel niedrigeren kumulativen Dosen zu einer irreversiblen Ovarialinsuffizienz kommen kann [35]. Dies stellt eine teilweise Abweichung vom reinen T2T-Konzept dar, scheint aber aufgrund praktischer Empfehlungen zur Behandlung des SLE sinnvoll zu sein. Demgegenüber ist es unter Umständen problematisch, dass der LLDAS nicht zugelassene Therapien ebenfalls ausschließt [26], zu denen in vielen Ländern nicht nur Rituximab, sondern auch Methotrexat zählt.

Wichtig ist, dass höhere Glukokortikoiddosen gemäß LLDAS und DORIS nicht zulässig sind - dies ist ein bedeutsamer Unterschied zur Remission bei der RA. Aus theoretischen Gründen könnte man einen solchen Ausschluss in einem T2T-Setting ebenfalls in Frage stellen. Allerdings ist das Erreichen einer niedrigen Krank- heitsaktivität oder sogar einer Remission durch eine Erhöhung der Glukokortikoiddosis auf mehr als 7,5 mg Predniso(lo)n pro Tag immer noch mit einem bedeutsamen Risiko für Tod, schwere Infektionen, kardiovaskuläre Erkrankungen, Osteoporose und andere Schäden verbunden [20, 36-38]. Zum Teil ist diese Assoziation auf einen Zuordnungsfehler (attribution bias) zurückzuführen, weil Patienten mit schwerem SLE in der Regel über längere Zeit höhere Dosen von Glukokortikoiden erhalten, doch weisen immer noch zahlreiche Datensätze auf die Risiken eines iatrogenen Cushing-Syndroms hin. Auf der anderen Seite sind 7,5 mg täglich zwar sicherer als höhere Dosierungen, und $5 \mathrm{mg}$ täglich erhöhen die Sicherheit noch weiter, doch liegen keine Daten vor, die überzeugend zeigen, dass irgendeine Glukokortikoiddosis langfristig vollständig sicher ist.

Infolgedessen fordern die T2T-Empfehlungen zu SLE ausdrücklich, dass die «SLE-Erhaltungstherapie die niedrigste zur Kontrolle der Krankheit erforderliche Glukokortikoid-Dosis anstreben sollte, und dass die Glukokortikoide möglichst vollständig abgesetzt werden sollten». Es überrascht nicht, dass diese Empfehlung ein hohes Maß an Zustimmung [9,6] erreichte. Zwar wurde argumentiert, dass dies eine gewisse, nicht gerechtfertigte Einseitigkeit gegenüber einem Medikament darstellt, das für die Behandlung der meisten SLE-Patienten immer noch wichtig ist, doch entspricht diese Empfehlung im vollen Umfang den jüngsten EULAR-Empfehlungen für das SLE-Management.

Das Problem des Ausschleichens der Glukokortikoide ist noch immer nicht komplett gelöst. Das vollständige Ausschleichen der oralen Glukokortikoide wird durch eine Hypothese erschwert, nach der das Absetzen niedriger Dosierungen von Glukokortikoiden auch nach Jahrzehnten, in denen die Krankheit gut kontrolliert war, das Risiko schwerer, organbedrohender Krankheitsschübe in relevantem Maß erhöht. Es liegen nur begrenzte Hinweise vor - die zudem überwiegend auf beobachteten Einzelfällen basieren -, dass dies bei der regelmäßigen Anwendung von Antimalariamitteln ein bedeutsames Problem darstellt. Tatsächlich konnte gezeigt werden, dass bei einem großen Teil der SLE-Patienten die Glukokortikoide abgesetzt werden können [39]. Trotzdem wurden gelegentlich Schübe festgestellt, darunter auch schwere, die Nieren betreffende Schübe. Während eine Senkung der Glukokortikoiddosis auf $\leq 5 \mathrm{mg}$ Predniso(lo)n pro Tag für die meisten Patienten sinnvoll erscheint, um das Langzeitergebnis zu verbessern, erfordert das vollständige Absetzen der Glukokortikoide unter Umständen ein vorsichtigeres, individuelles Vorgehen.

Die kombinierten Ziele einer Remission bei möglichst niedrigen Glukokortikoiddosierungen laufen auf einen verstärkten Einsatz von DMARDs und Immunsuppressiva hinaus. Dies wird zum Teil dadurch erschwert, dass es weiterhin nur eine geringe Zahl solcher Medikamente gibt, die für die Behandlung des SLE zugelassen sind, und die sich, neben Antimalariamitteln und Glukokortikoiden, im Wesentlichen auf Azathioprin und den AntiBLyS/BAFF-Antikörper Belimumab beschränken [15, 40], auch wenn Mycophenolat und Methotrexat in vielen Ländern akzeptiert werden. Dennoch sind die verfügbaren Medikamente schon heute oftmals ausreichend, um die Krankheit zu kontrollieren,
48

Kompass Autoimmun 2020;2:45-5

DOI: $10.1159 / 000507644$ 


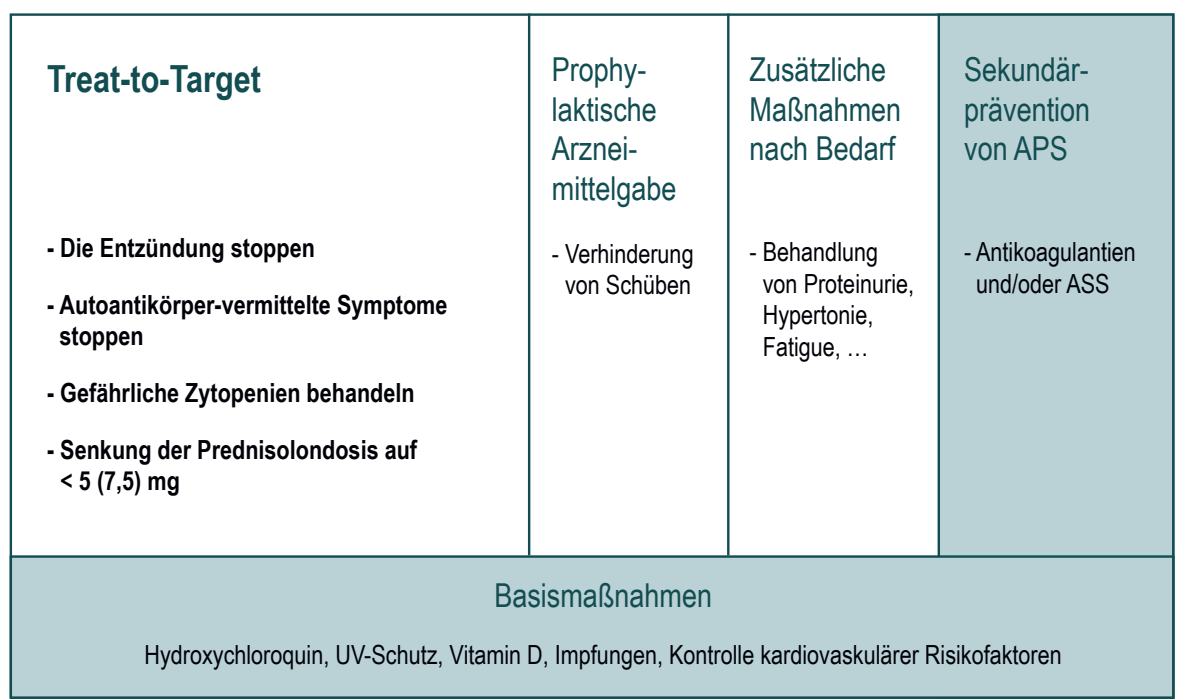

Abb. 1. Der Treat-to-Target-Ansatz bei SLE erfordert zusätzliche Elemente, um das adäquate SLEPatientenmanagement vollständig abzubilden. seprophylaxe mit Vitamin D, Influenza- und Pneumokokkenimpfung sowie das Management von Risikofaktoren für Atherosklerose $[45,46]$. Das Management von Risikofaktoren beinhaltet vor allem die Hypertoniekontrolle, insbesondere bei Patienten mit Nierenbeteiligung [3, 32]. Als Mittel der ersten Wahl werden vornehmlich ACE-Hemmer oder Angiotensin-Rezeptorblocker empfohlen, die auch eine wichtige Rolle bei der Verringerung der Proteinurie und der Erhaltung der Nierenfunktion spielen. Um alle Ansätze, die das Immunsystem nicht direkt beeinflussen, zu berücksichtigen, heißt es in den T2T-Empfehlungen allgemein, dass «relevante Therapien, die die Immunmodulation ergänzen, erwogen werden sollten, um die Komorbidität bei SLE-Patienten zu kontrollieren» [3]. Tatsächlich sind solche Maßnahmen oftmals genauso wichtig für die Morbidität und Mortalität wie die immunmodulatorische Behandlung.

Aus ähnlichen Überlegungen wird in den T2T-Empfehlungen anerkannt, dass SLE-Patienten auch unter Symptomen leiden, die nicht durch die Krankheitsaktivität des SLE, sondern durch Organschäden und verschiedene andere Faktoren, darunter auch psychologische und umweltbedingte Einflüsse, verursacht werden. Dementsprechend erklären die Empfehlungen «Faktoren, die die gesundheitsbezogene Lebensqualität (health-related quality of life, HRQoL) negativ beeinflussen, wie Fatigue, Schmerzen und Depression, sollten zusätzlich zur Kontrolle der Krankheitsaktivität behandelt werden, um einen Schaden zu verhindern.» Diese Faktoren, die häufig vorliegen [22, 23], sind mit einer starken Beeinträchtigung der HRQoL verbunden und haben negative Auswirkungen auf die Prognose.

Aus Gründen der Vollständigkeit sollten prophylaktische Ansätze auch die überwiegend sekundäre Prävention bei APS beinhalten. Zwar hat etwa die Hälfte der APS-Patienten auch einen SLE, doch spricht diese Seite der Erkrankung nicht auf eine Immunsuppression an, sondern erfordert die Gabe von Antikoagulantien und/ oder niedrig dosierter Acetylsalicylsäure (ASS). In den T2T-Empfehlungen heißt es entsprechend: «Die Prävention und Behandlung der Morbidität durch ein sekundäres APS sollte ein SLE-The-

$\mathrm{Zu}$ den weiteren prophylaktischen Maßnahmen, die in diesem Stadium eingeleitet werden, gehören Sonnenschutz, Osteoporo- 
Table 1. Versuch, die Kernpunkte der Treat-to-Target-Empfehlungen für SLE herauszuarbeiten

Übergeordnete Prinzipien

- Diegemeinsame Entscheidungsfindung ist von zentraler Bedeutung

- Bekämpfung von Tod, Schaden und geringer Lebensqualität durch Kontrolle der SLE-Aktivität und Arzneimitteltoxizität.

- $\quad$ Das SLE-Management erfordert ein (multidisziplinäres) Verständnis des SLE.

- $\quad$ SLE-Patienten müssen überwacht werden, auch wenn es ihnen gut geht.

Empfehlungen

Die Remission (oder geringe Krankheitsaktivität) zum Ziel machen. Schübe verhindern.

Keine reine Serologie behandeln.

Organschäden verhindern.

Eine Verbesserung der Lebensqualität über die SLE-Aktivität hinaus anstreben

Die Lupusnephritis frühzeitig diagnostizieren und behandeln.

Jede Lupusnephritis mindestens 3 Jahre behandeln

Glukokortikoide so weit wie möglich ausschleichen.

Das sekundäre Anti-Phospholipid-Syndrom mit Antikoagulantien/

ASS behandeln.

Konsequenter Einsatz von Antimalariamitteln.

Begleitmedikation über die Immunmodulation hinaus ist wichtig.

rapieziel sein; die therapeutischen Empfehlungen unterscheiden sich nicht von denen beim primären APS» [3]. Der einzige Unterschied besteht darin, dass bei SLE-assoziiertem APS zusätzlich Antimalariamittel empfohlen werden, eine Empfehlung, die bei Patienten mit primärem APS nicht so eindeutig ist [44].

\section{Schlussfolgerungen}

Es erscheint lohnenswert, dem T2T-Ansatz zu folgen, um das Behandlungsergebnis von Patienten mit SLE zu verbessern. Tatsächlich gibt es jetzt zumindest ungefähr definierte Ziele sowie konsensbasierte Empfehlungen für diesen Ansatz. Zwar fußt der T2TAnsatz beim SLE auf den gleichen Grundsätzen wie bei RA [47] oder Spondyloarthritiden [48], doch sind für den SLE einige Veränderungen erforderlich, um die entscheidenden Schritte in der Behandlung von SLE-Patienten vollständig abzubilden (Abb. 1). Zum einen wird eine Senkung der Prednisolon-Äquivalenzdosen auf höchstens 5 mg empfohlen, und längerfristig sollte die Dosierung nicht mehr als 7,5 mg betragen, um schwerwiegende Folgen zu vermeiden. Wegen der schweren Toxizität, die mit höheren kumulativen Dosen verbunden ist, gilt die Dosisrestriktion noch mehr für Cyclophosphamid. Zum zweiten haben die Basismaßnahmen Antimalariamittel, UV-Schutz, Vitamin D, Impfungen und die Kontrolle der kardiovaskulären Risikofaktoren so grundlegenden Einfluss auf die Langzeitergebnisse, dass sie bei jedem SLE-Patienten unabhängig von der Therapie eingeleitet werden sollten. Zum Dritten erfordern einige Formen der Organbeteiligung eine medikamentöse Behandlung über die immunmodulatorische Therapie hinaus, wie beispielsweise die Gabe von ACEHemmern oder Angiotensin-Rezeptorblockern bei Proteinurie. Desgleichen ist auch bei Fatigue, Schmerzen, Depressionen und anderen relevanten Symptomen eine Behandlung erforderlich, die über die Immunmodulation hinausgeht, auch wenn keine SLE-Krankheitsaktivität vorliegt. Und viertens wird ein APS nicht mittels Immunsuppressiva, sondern mit Antikoagulantien und/oder Thrombozytenaggregationshemmern behandelt.

Abgesehen davon ist es entscheidend, bei jeder Visite die Entzündungssymptome durch klinische und laborchemische Untersuchungen zu erkennen und entsprechend zu behandeln. Daher empfiehlt es sich, zumindest die einzelnen Organsymptome eines validierten SLE-Krankheitsaktivitätsscores abzufragen und ein großes Blutbild (auf Zytopenien) und eine Urinuntersuchung (auf Protein) durchzuführen sowie Entzündungsparameter und Komplementverbrauch zu messen [49]. Es muss ein Stopp jeglicher SLE-bedingter Entzündungsaktivität erreicht werden. Dies erfolgt normalerweise durch Gabe von DMARDs und/oder Immunsuppressiva, zunächst in Kombination mit Glukokortikoiden, die anschließend auf niedrigere Dosierungen verringert werden. Das gleiche Prinzip gilt auch für relevante antikörpervermittelte Symptome wie die Lupus-Psychose oder kritische Zytopenien. Es liegen mittlerweile Studienergebnisse vor, dass ein solcher Ansatz sowohl umsetzbar als auch wirksam ist (Tab. 1).

\section{Interessenkonflikte}

M. A.: Advisory Boards und/oder Vorträge für Astra Zeneca, GSK, Lilly, Janssen, Pfizer, Roche.

N. L.: Advisory Boards und/oder Vorträge für AbbVie, Boehringer-Ingelheim, Janssen, Novartis, Pfizer, Roche, Sanofi-Aventis, UCB.

M. S.: Advisory Boards und/oder Vorträge für Abbvie; Astra-Zeneca; Biogen; Boehringer-Ingelheim; BMS; Celgene; Chugai; GSK; Janssen-Cilag; Lilly; MSD; Novartis; Pfizer; Protagen; Roche; Sanofi-Aventis; UCB; zudem erhielt er Forschungsgelder von Abbvie, GSK und UCB.

\section{Literatur}

-1 Aringer M, Dorner T, Leuchten N, et al.: Toward new criteria for systemic lupus erythematosus-a standpoint. Lupus 2016;25:805-811.

-2 van VR, Voskuyl A, Bertsias G, et al.: A framework for remission in SLE: consensus findings from a large international task force on definitions of remission in SLE (DORIS). Ann Rheum Dis 2017;76:554-561.

-3 van Vollenhoven RF, Mosca M, Bertsias G, et al.: Treat-to-target in systemic lupus erythematosus: recommendations from an international task force. Ann Rheum Dis 2014;73:958-967.
4 Smolen JS, Aletaha D, Bijlsma JW, et al.: Treating rheumatoid arthritis to target: recommendations of an international task force. Ann Rheum Dis 2010;69:631-637.

5 Smolen JS, Landewe R, Bijlsma J, et al.: EULAR recommendations for the management of rheumatoid arthritis with synthetic and biological disease-modifying antirheumatic drugs: 2016 update. Ann Rheum Dis 2017;76: 960-977.

6 Rahman A, Isenberg DA: Systemic lupus erythematosus. N Engl J Med 2008;358:929-939.
7 Tsokos GC: Systemic lupus erythematosus. N Engl J Med 2011;365:2110-2121.

${ }_{8}$ Bencivelli W, Vitali C, Isenberg DA, et al.: Disease activity in systemic lupus erythematosus: report of the Consensus Study Group of the European Workshop for Rheumatology Research. III. Development of a computerised clinical chart and its application to the comparison of different indices of disease activity. The European Consensus Study Group for Disease Activity in SLE. Clin Exp Rheumatol 1992;10:549-554 
$\$ 9$ Yee CS, Farewell VT, Isenberg DA, et al.: The use of Systemic Lupus Erythematosus Disease Activity Index-2000 to define active disease and minimal clinically meaningful change based on data from a large cohort of systemic lupus erythematosus patients. Rheumatology (Oxford) 2011;50:982-988.

-10 Touma Z, Gladman DD, Su J, et al.: A novel lupus activity index accounting for glucocorticoids: SLEDAI-2K glucocorticoid index. Rheumatology (Oxford) 2018;57:1370-1376.

11 Jesus D, Matos A, Henriques C, et al.: Derivation and validation of the SLE Disease Activity Score (SLE-DAS): a new SLE continuous measure with high sensitivity for changes in disease activity. Ann Rheum Dis 2019;78:365-371.

12 Steinberg AD, Steinberg SC: Long-term preservation of renal function in patients with lupus nephritis receiving treatment that includes cyclophosphamide versus those treated with prednisone only. Arthritis Rheum 1991; 34:945-950.

13 Bertsias GK, Ioannidis JP, Aringer M, et al. EULAR recommendations for the management of systemic lupus erythematosus with neuropsychiatric manifestations: report of a task force of the EULAR standing committee for clinical affairs. Ann Rheum Dis 2010;69: 2074-2082.

14 Bertsias G, Ioannidis JP, Boletis J, et al.: EULAR recommendations for the management of systemic lupus erythematosus. Report of a Task Force of the EULAR Standing Committee for International Clinical Studies Including Therapeutics. Ann Rheum Dis 2008 67:195-205

15 Aringer M, Burkhardt H, Burmester GR, et al. Current state of evidence on 'off-label' therapeutic options for systemic lupus erythematosus, including biological immunosuppressive agents, in Germany, Austria and Switzerland a consensus report. Lupus 2012;21:386-401.

-16 Illei GG, Takada K, Parkin D, et al.: Renal flares are common in patients with severe proliferative lupus nephritis treated with pulse immunosuppressive therapy: long-term followup of a cohort of 145 patients participating in randomized controlled studies. Arthritis Rheum 2002;46:995-1002.

17 Stoll T, Sutcliffe N, Mach J, et al.: Analysis of the relationship between disease activity and damage in patients with systemic lupus erythematosus - a 5-yr prospective study. Rheumatology (Oxford) 2004;43:1039-1044.

18 Lopez R, Davidson JE, Beeby MD, et al.: Lupus disease activity and the risk of subsequent organ damage and mortality in a large lupus cohort. Rheumatology (Oxford) 2012;51:491-498.

19 Roman MJ, Shanker BA, Davis A, et al.: Prevalence and correlates of accelerated atherosclerosis in systemic lupus erythematosus. N Engl J Med 2003;349:2399-2406.

-20 Bessant R, Duncan R, Ambler G, et al.: Prevalence of conventional and lupus-specific risk factors for cardiovascular disease in patients with systemic lupus erythematosus: A case-control study. Arthritis Rheum 2006;55:892-899.

-21 Franklyn K, Hoi A, Nikpour M, et al.: The need to define treatment goals for systemic lupus erythematosus. Nat Rev Rheumatol 2014;10:567-571.
22 Leuchten N, Bauernfeind B, Kuttner J, et al.: Relevant concepts of functioning for patients with systemic lupus erythematosus identified in a Delphi exercise of experts and a literature review. Arthritis Care Res (Hoboken ) 2014;66: 1895-1904.

23 Leuchten N, Milke B, Winkler-Rohlfing B, et al.: Early symptoms of systemic lupus erythematosus (SLE) recalled by 339 SLE patients. Lupus 2018;27:1431-1436.

24 van Vollenhoven RF, Petri MA, Cervera R, et al.: Belimumab in the treatment of systemic lupus erythematosus: high disease activity predictors of response. Ann Rheum Dis 2012;71: 1343-1349.

25 Steiman AJ, Gladman DD, Ibanez D, et al.: Outcomes in patients with systemic lupus erythematosus with and without a prolonged serologically active clinically quiescent period. Arthritis Care Res (Hoboken ) 2012;64: 511-518.

26 Franklyn K, Lau CS, Navarra SV, et al.: Definition and initial validation of a Lupus Low Disease Activity State (LLDAS). Ann Rheum Dis 2016;75:1615-1621.

27 Morand EF, Trasieva T, Berglind A, et al.: Lupus Low Disease Activity State (LLDAS) attainment discriminates responders in a systemic lupus erythematosus trial: post-hoc analysis of the Phase IIb MUSE trial of anifrolumab. Ann Rheum Dis 2018;77:706-713.

28 Oon S, Huq M, Golder V, et al.: Lupus Low Disease Activity State (LLDAS) discriminates responders in the BLISS-52 and BLISS-76 phase III trials of belimumab in systemic lupus erythematosus. Ann Rheum Dis 2019.

29 Petri M, Magder LS: Comparison of Remission and Lupus Low Disease Activity State in Damage Prevention in a United States Systemic Lupus Erythematosus Cohort. Arthritis Rheumatol 2018;70:1790-1795.

30 Golder V, Huq M, Franklyn K, et al.: Does expert opinion match the operational definition of the Lupus Low Disease Activity State (LLDAS)? A case-based construct validity study. Semin Arthritis Rheum 2017;46: 798-803.

31 Tani C, Vagelli R, Stagnaro C, et al.: Remission and low disease activity in systemic lupus erythematosus: an achievable goal even with fewer steroids? Real-life data from a monocentric cohort. Lupus Sci Med 2018;5:e000234.

32 Bertsias GK, Tektonidou M, Amoura Z, et al.: Joint European League Against Rheumatism and European Renal Association-European Dialysis and Transplant Association (EULAR/ ERA-EDTA) recommendations for the management of adult and paediatric lupus nephritis. Ann Rheum Dis 2012;71:1771-1782.

33 Alexander T, Thiel A, Rosen O, et al.: Depletion of autoreactive immunologic memory followed by autologous hematopoietic stem cell transplantation in patients with refractory SLE induces long-term remission through de novo generation of a juvenile and tolerant immune system. Blood 2009;113:214-223.

34 Zen M, Iaccarino L, Gatto M, et al.: Lupus low disease activity state is associated with a decrease in damage progression in Caucasian patients with SLE, but overlaps with remission. Ann Rheum Dis 2018;77:104-110.
5 Boumpas DT, Austin HA, III, Vaughan EM, et al.: Risk for sustained amenorrhea in patients with systemic lupus erythematosus receiving intermittent pulse cyclophosphamide therapy. Ann Intern Med 1993;119:366-369.

36 Huscher D, Thiele K, Gromnica-Ihle E, et al.: Dose-related patterns of glucocorticoid-induced side effects. Ann Rheum Dis 2009;68: 1119-1124.

37 Gonzalez-Echavarri C, Capdevila O, Espinosa $\mathrm{G}$, et al.: Infections in newly diagnosed Spanish patients with systemic lupus erythematosus: data from the RELES cohort. Lupus 2018; 27:2253-2261.

38 Chen HL, Shen LJ, Hsu PN, et al.: Cumulative Burden of Glucocorticoid-related Adverse Events in Patients with Systemic Lupus Erythematosus: Findings from a 12-year Longitudinal Study. J Rheumatol 2018;45:83-89.

39 Tani C, Carli L, Vagelli R, et al.: Glucocorticoids withdrawal in systemic lupuserythematosus: myth or reality? Ann Rheum Dis 2016 75(Suppl2):290. (Abstract)

40 Navarra SV, Guzman RM, Gallacher AE, et al.: Efficacy and safety of belimumab in patients with active systemic lupus erythematosus: a randomised, placebo-controlled, phase 3 trial. Lancet 2011;377:721-731.

41 Stohl W, Schwarting A, Okada M, et al.: Efficacy and safety of subcutaneous belimumab in systemic lupus erythematosus: a fifty-two-week randomized, double-blind, placebo-controlled study. Arthritis Rheumatol 2017;69:1016-1027.

42 A randomized study of the effect of withdrawing hydroxychloroquine sulfate in systemic lupus erythematosus. The Canadian Hydroxychloroquine Study Group. N Engl J Med 1991; 324:150-154.

43 Ruiz-Irastorza G, Ramos-Casals M, Brito-Zeron P, et al.: Clinical efficacy and side effects of antimalarials in systemic lupus erythematosus: a systematic review. Ann Rheum Dis 2010;69: 20-28.

44 Andreoli L, Bertsias GK, Agmon-Levin N, et al.: EULAR recommendations for women's health and the management of family planning, assisted reproduction, pregnancy and menopause in patients with systemic lupus erythematosus and/or antiphospholipid syndrome. Ann Rheum Dis 2017;76:476-485.

45 Aringer M, Schneider M. [Recipes systemic lupus erythematosus.]. Z Rheumatol 2014;73: 496-500.

46 Schneider M: Target therapy in sle. Autoimmun Rev 2019;18:21-24

47 Smolen JS, Breedveld FC, Burmester GR, et al.: Treating rheumatoid arthritis to target: 2014 update of the recommendations of an international task force. Ann Rheum Dis 2016;75:3-15.

48 Smolen JS, Schols M, Braun J, et al.: Treating axial spondyloarthritis and peripheral spondyloarthritis, especially psoriatic arthritis, to target: 2017 update of recommendations by an international task force. Ann Rheum Dis 2018; 77:3-17.

49 Mosca M, Tani C, Aringer M, et al.: European League Against Rheumatism recommendations for monitoring patients with systemic lupus erythematosus in clinical practice and in observational studies. Ann Rheum Dis 2010; 69:1269-1274. 